Article

\title{
Characterization of FLOWERING LOCUS C Homologs in Apple as a Model for Fruit Trees
}

\author{
Hidenao Kagaya ${ }^{1}$, Naoko Ito ${ }^{1}$, Tomoki Shibuya ${ }^{2}$, Sadao Komori ${ }^{3}$, Kazuhisa Kato ${ }^{1, *}$ and \\ Yoshinori Kanayama $1, *(\mathbb{D}$ \\ 1 Graduate School of Agricultural Science, Tohoku University, Aoba-ku, Sendai 980-8572, Japan \\ 2 Faculty of Life and Environmental Science, Shimane University, Matsue 690-8504, Japan \\ 3 Faculty of Agriculture, Iwate University, Morioka 020-8550, Japan \\ * Correspondence: kazuhisa.kato.d8@tohoku.ac.jp (K.K.); yoshinori.kanayama.a7@tohoku.ac.jp (Y.K.)
}

Received: 28 April 2020; Accepted: 24 June 2020; Published: 26 June 2020

\begin{abstract}
To elucidate the molecular mechanism of juvenility and annual flowering of fruit trees, FLOWERING LOCUS C (FLC), an integrator of flowering signals, was investigated in apple as a model. We performed sequence and expression analyses and transgenic experiments related to juvenility with annual flowering to characterize the apple $F L C$ homologs MdFLC. The phylogenetic tree analysis, which included other MADS-box genes, showed that both MdFLC1 and MdFLC3 belong to the same FLC group. MdFLC1c from one of the MdFLC1 splice variants and MdFLC3 contain the four conserved motives of an MIKC-type MADS protein. The mRNA of variants MdFLC1a and MdFLC1b contain intron sequences, and their deduced amino acid sequences lack $\mathrm{K}$ - and $\mathrm{C}$-domains. The expression levels of MdFLC1a, MdFLC1b, and MdFLC1c decreased during the flowering induction period in a seasonal expression pattern in the adult trees, whereas the expression level of MdFLC 3 did not decrease during that period. This suggests that $M d F L C 1$ is involved in flowering induction in the annual growth cycle of adult trees. In apple seedlings, because phase change can be observed in individuals, seedlings can be used for analysis of expression during phase transition. The expression levels of $M d F L C 1 b, M d F L C 1 c$, and $M d F L C 3$ were high during the juvenile phase and low during the transitional and adult phases. Because the expression pattern of MdFLC3 suggests that it plays a specific role in juvenility, $M d F L C 3$ was subjected to functional analysis by transformation of Arabidopsis. The results revealed the function of $M d F L C 3$ as a floral repressor. In addition, MdFT had CArG box-like sequences, putative targets for the suppression of flowering by MdFLC binding, in the introns and promoter regions. These results indicate that apple homologs of FLC, which might play a role upstream of the flowering signals, could be involved in juvenility as well as in annual flowering. Apples with sufficient genome-related information are useful as a model for studying phenomena unique to woody plants such as juvenility and annual flowering.
\end{abstract}

Keywords: Malus domestica; Rosaceae; juvenility; FLOWERING LOCUS C; flowering

\section{Introduction}

Fruit trees, which are perennial woody plants, have a long juvenile period after germination and before flowering and fruit set. Because fruit quality cannot be evaluated during this juvenile phase, it is necessary to address the long juvenile period of fruit trees with regard to fruit tree breeding. Lengths of juvenile periods differ among species, and apple requires six to eight years of juvenility [1]. Although some cultivation techniques to shorten the juvenile period have been proposed, such as plant hormone treatment, grafting to dwarf rootstocks, suppression of dormancy, and adjustment of cultivation conditions [2-4], little is known about the molecular mechanisms of juvenility. It is more difficult to elucidate the mechanism underlying the control of juvenility in fruit trees than in 
herbaceous plants because fruit trees take longer to grow and are larger in size. Apple is one of the earliest plants whose genome sequences have been reported as woody plants and fruit tree [5], and is utilized as a model because of its extensive genome information [6,7]. Therefore, apples are useful for studying phenomena unique to woody plants such as juvenility and annual flowering.

In general, juvenility is stronger in young seedlings and gradually weakens as age progresses. There is also known to be a gradient of juvenility in individual trees; that is, juvenility is stronger at the base of trunks and branches and becomes weaker approaching the tip [8]. Genes that are potentially related to juvenility have been reported in studies of homologs of flowering-related genes from Arabidopsis, which is a model herbal plant. Previous studies focused on the relationship between juvenility and the apple homologs of TERMINAL FLOWER 1 (TFL1), FLOWERING LOCUS T (FT), $\operatorname{LEAFY}(L F Y)$, and APETALA1 (AP1), which are floral meristem identity-related genes in Arabidopsis. The expression of apple TFL1 homolog MdTFL is high during the juvenile phase, and the expression of apple $F T$ homolog MdFT is high during the adult phase [9]. The overexpression of apple homologs of $L F Y$ and AP1 in Arabidopsis promotes flowering, and the overexpression of MdTFL delays flowering [10-12]. In citrus, the overexpression of $L F Y$ or AP1 of Arabidopsis reportedly decreases the juvenile period from seven years to two years [13], and the methylation of the LEAFY homolog is thought to be involved in juvenility [14]. However, most of these genes are located relatively downstream of the flowering pathway, and in order to clarify the molecular mechanism of juvenility, it is necessary to analyze genes farther upstream.

There are several flowering pathways, including the photoperiod, vernalization, gibberellin, and autonomous pathways [15]. The photoperiod and vernalization-dependent pathways are controlled by environmental factors, and the gibberellin-dependent pathway is comprised of a group of genes related to the synthesis and signal transduction of gibberellin. In contrast, the autonomous pathway is dependent on endogenous growth-related factors. Juvenility is not reduced by environmental factors such as temperature and day length, but is reduced by growth over several years, which suggests that juvenility is controlled by endogenous growth-related factors. Some genes in the autonomous pathway are known to induce flowering by suppressing the expression of FLOWERING LOCUS C (FLC) [16]. FLC plays a role as a key regulator of the autonomous and vernalization pathways and inhibits flowering by suppressing the expression of floral induction genes SOC1 and FT in Arabidopsis [17-19]. Since FLC is a key gene for the flowering pathways, including the autonomous pathway, it can be expected to play a role in the suppression of flowering in the juvenile phase.

There have been some reports on FLC homologs in fruit trees. FLC homologs have been identified in apple, and divergent functions have been suggested based on their nucleotide sequences [20]. Two of these exhibit increased expression during dormancy and decreased expression with dormancy release, which suggests that they repress flowering as described in Arabidopsis. In fact, the possibility that the $F L C$-like gene is a candidate gene is mentioned in the genome-wide association mapping of the flowering period in apple [21]. In other apple FLC-like genes, the repression of bud outgrowth during dormancy and the promotion of flowering under non-chilling conditions have been reported [22,23]. Among other Rosaceae fruit trees, the FLC homolog in peach (Prunus persica) has not been reported to be associated with dormancy, and its role has not been elucidated [24]. No FLC homolog has been found in Japanese apricot (Prunus mume) either [25]. In fruit trees other than Rosaceae, changes in the expression and splicing of an FLC-like gene were reported in trifoliate orange (Poncirus trifoliata) [26]. Although these reports provide some information about the role of $F L C$ in fruit trees, information about the relationship between juvenility and $F L C$ homologs is still lacking. Therefore, to investigate the physiological roles of apple $F L C$ homolog $M d F L C$ at the molecular level, sequence and expression analyses and transgenic experiments were performed. 


\section{Results}

\section{1. cDNA Isolation and Phylogenetic Tree Analysis of MdFLC}

Nine apple expressed sequence tag (EST) sequences were obtained by a BLAST search in DNA Data Bank of Japan (DDBJ, http://blast.ddbj.nig.ac.jp) using the amino acid sequence of the MADS region of Arabidopsis FLC. The contig sequences corresponding to these EST sequences were searched in GDR (https://www.rosaceae.org), a Rosaceae genome database, to obtain four contig sequences. Six EST or contig sequences remained after excluding duplicate sequences. A phylogenetic tree was prepared based on the amino acid sequences of these six genes and MADS-box genes from Arabidopsis and apple, and three of the six sequences were assigned to the same group as Arabidopsis FLC. Next, one of the three sequences was detected by PCR using cDNA from the juvenile phase of apple seedlings with primers specific to the three sequences and designated as MdFLC1 (MD05G1037100) [23]. Three kinds of mRNA sequence were obtained by the RACE method and designated as splice variants $M d F L C 1 a$ (accession number LC550081), MdFLC1b (LC550082), and MdFLC1c (LC550083).

PCR was performed using cDNA from the juvenile phase of apple seedlings with degenerate primers in the MADS region, and 10 kinds of MADS box-like sequences were obtained. One sequence among them was found to be juvenile phase-specific and homologous to Arabidopsis FLC; it was designated as MdFLC3 (MD10G1041100) [23].

Figure 1 shows the amino acid sequence alignment of MdFLC1, MdFLC3, and AtFLC (AF537203) from Arabidopsis. MdFLC1c, which was the longest of the MdFLC1 mRNA variants, and MdFLC3 contained MADS-, K-, I-, and C-domains with AtFLC. A phylogenetic tree of the amino acid sequence of MdFLC1c, MdFLC3, and FLC homologous proteins from other plants was prepared with other MADS-box proteins based on [27] (Figure 2). FLC, SVP, SOC1, AP1, and SEP groups were formed, and MdFLC1c and MdFLC3 were included in the FLC group.

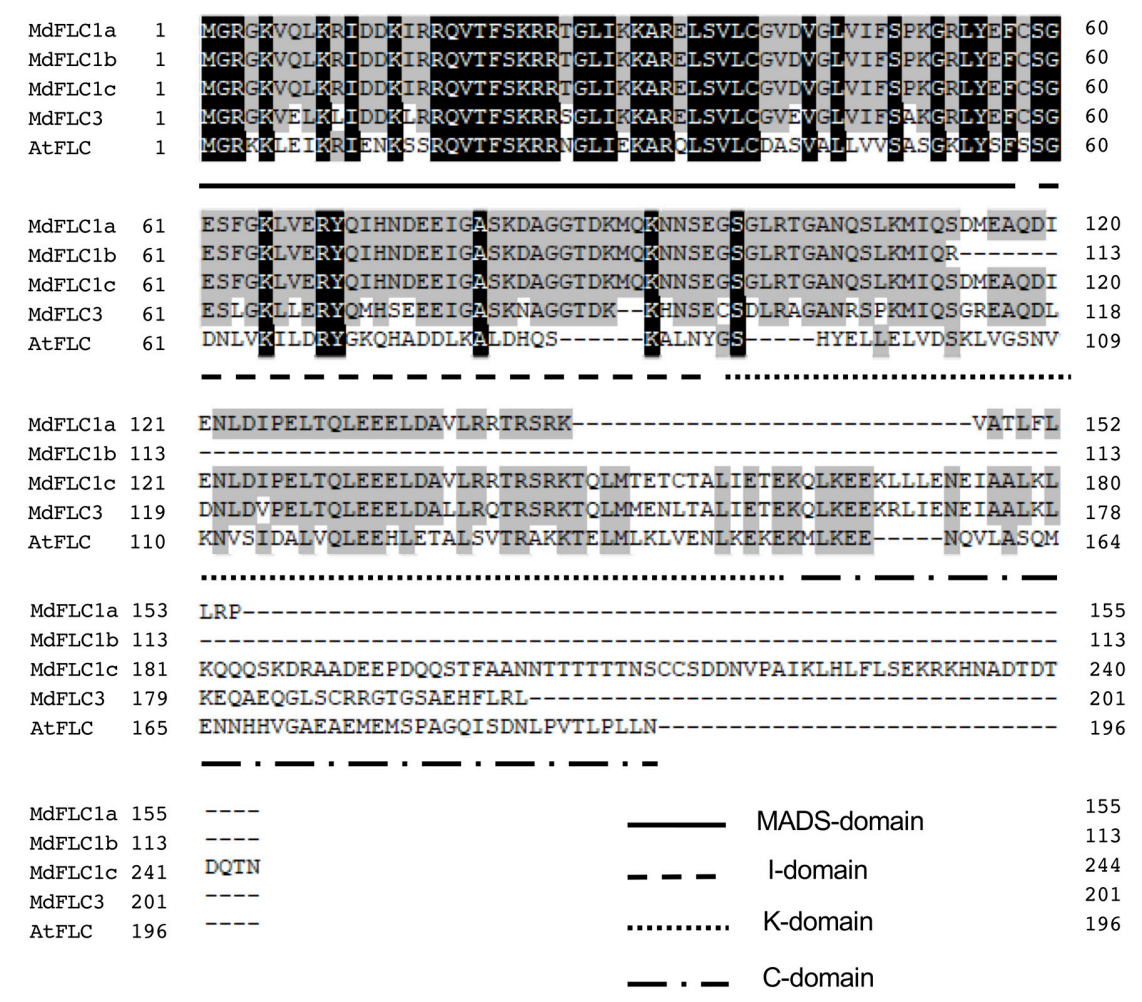

Figure 1. Amino acid sequence alignment of MdFLC and AtFLC. The MADS-, K-, I-, and C-domains are underlined. Identical amino acids for five and less proteins are shown in black and gray boxes, respectively. 


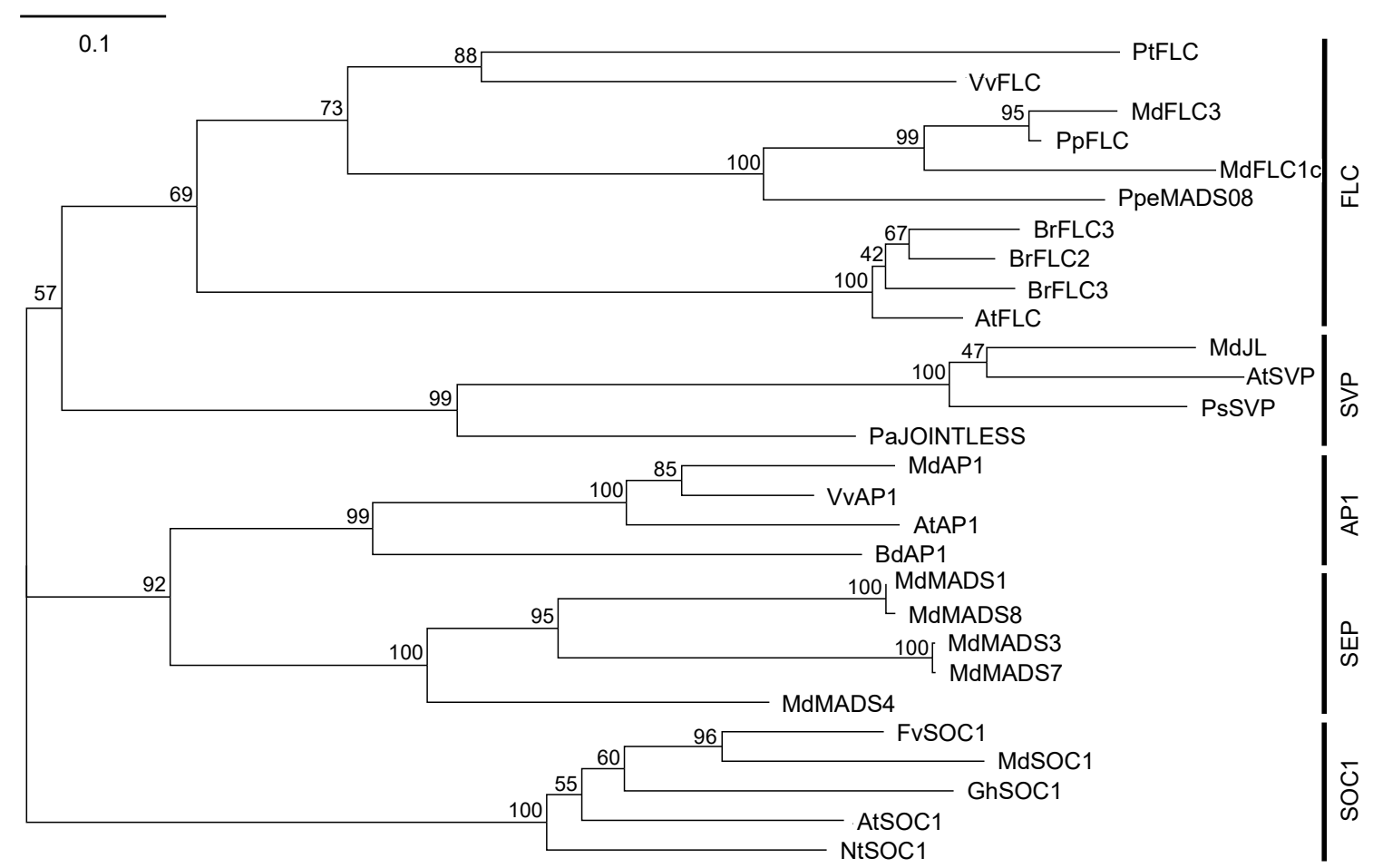

Figure 2. A phylogenetic tree based on the amino acid sequences of FLC, SVP, AP1, SEP, and SOC1 homologs from various species. The tree was constructed by the neighbor-joining method after sequence alignment using the ClustalW program. Branch numbers refer to percentage of replicates that support the branch using the bootstrap method (1000 replicates). The scale bar corresponds to 0.1 amino acid substitutions per residue. The accession numbers of the proteins added to construct the phylogenetic tree are as follows: PtFLC (EU497676), VvFLC (GU133630), PpFLC (KP164015), BrFLC1 (DQ866874), BrFLC2, (DQ866875), BrFLC3 (DQ866876), MdJOINTLESS (DQ402055), AtSVP (AF211171), PsSVP (AY830919), PaJOINTLESS (EU332978), VvAP1 (GU133634), MdAP1 (EU672877), AtAP1 (BT004113), BdAP1 (HQ588324), MdMADS1 (U78947), MdMADS3 (U78949), MdMADS4 (U78950), MdMADS7 (AJ000760), MdMADS8 (AJ001681), FvSOC1 (FJ531999), MdSOC1 (DQ846833), GhSOC1 (JF701982), NtSOC1 (JQ686938), AtSOC1 (AY093967).

\subsection{Expression Analysis of MdFLC in the Adult Trees}

Seasonal changes in the expression of MdFLC were examined in the adult trees. Flowering induction occurs between late June and mid-July [27]. The expression levels of MdFLC1a, MdFLC1b, and MdFLC1c were high in early June and decreased in early July during the period of flowering induction (Figure 3a-c). On the other hand, the expression level of MdFLC3 did not change from early June to early July, but increased in August (Figure 3d). FLC suppresses the expression of FT in leaves as mentioned above [17]. FT generally produces mobile floral signals in leaves [28], and FT signal movement is also reported in Rosaceae fruit trees [29]. Therefore, leaves were used for analysis in the present study. 

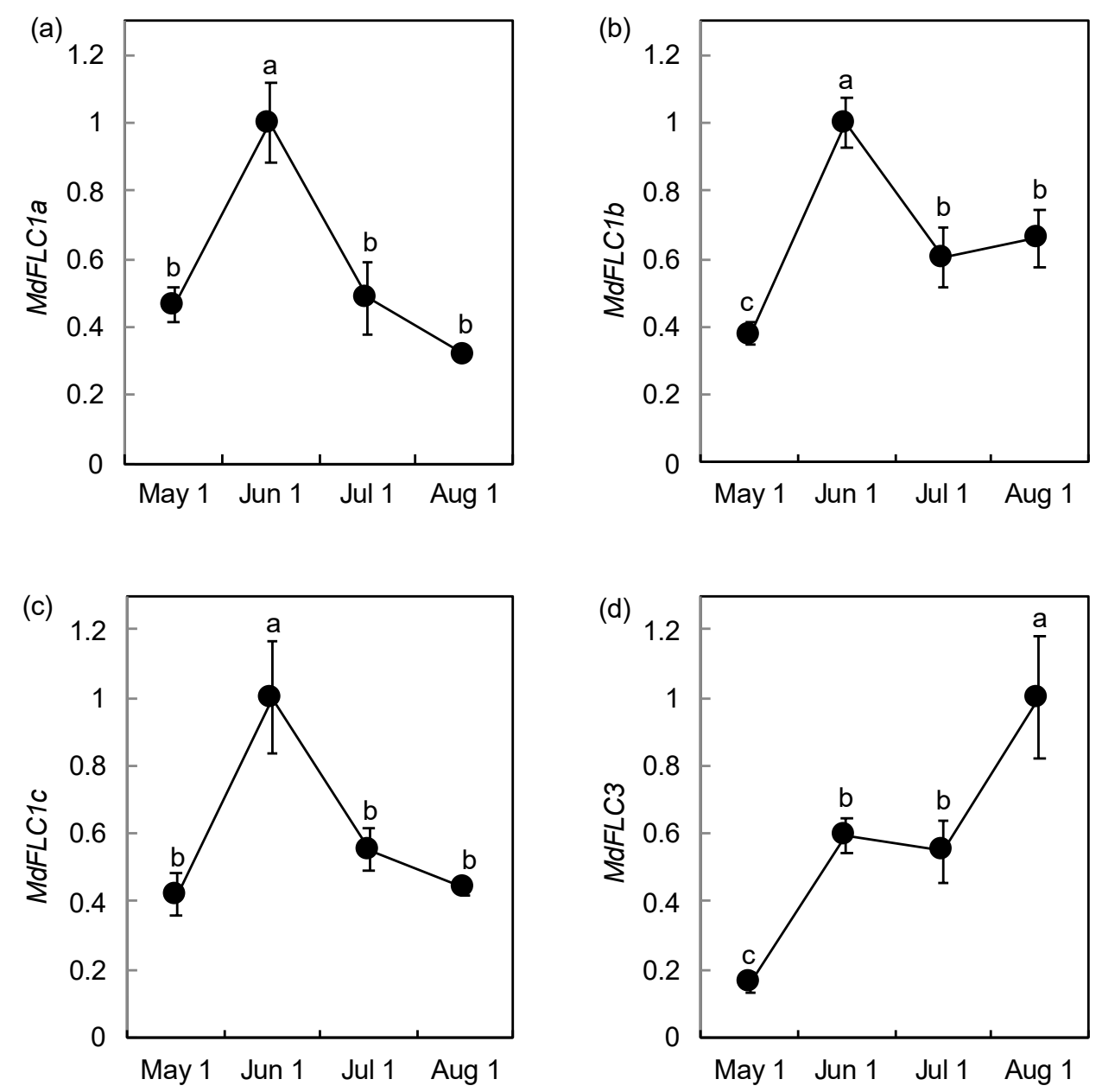

Figure 3. Seasonal changes in the expression levels of MdFLC1a (a), MdFLC1b (b), $M d F L C 1 c(\mathbf{c})$, and MdFLC3 (d) in the leaves of adult trees. Total RNA was prepared for the expression analysis of MdFLC1 and MdFLC3 on May 1, June 1, July 1, and August 1, 2016. Relative expression was determined using triplicate measurements taken from three independent biological replicates. The relative expression levels were normalized against MdACTIN with standard errors, and the maximum level of the transcripts was set at 1.0. The values with different letters for each gene significantly differed between days at $p<0.05$, according to a Tukey test.

\subsection{Expression Analysis of MdFLC During Phase Transition}

The expression level of $M d F L C$ during phase transition was performed in apple seedlings. While MdFLC1a expression was not detectable, the expression levels of MdFLC1b and MdFLC1c were high during the juvenile phase and low during the transitional and adult phases (Figure 4). The expression level of MdFLC3 during the juvenile phase was also high compared to that in the transitional and adult phases, and it was 7.4 times the level in the adult phase. 
Int. J. Mol. Sci. 2020, 21, 4562

6 of 13

(a)

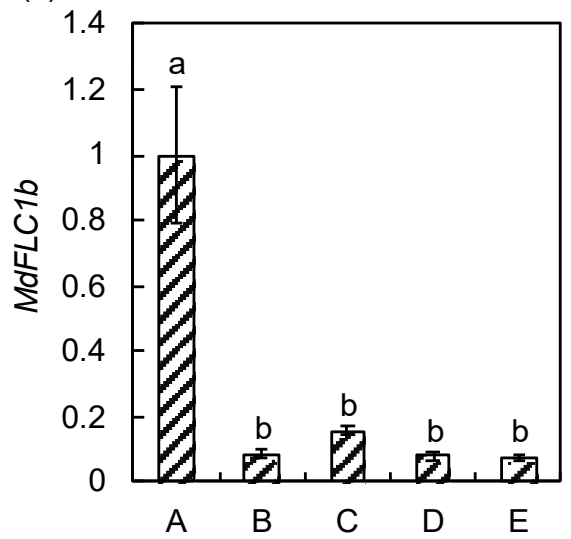

(b)

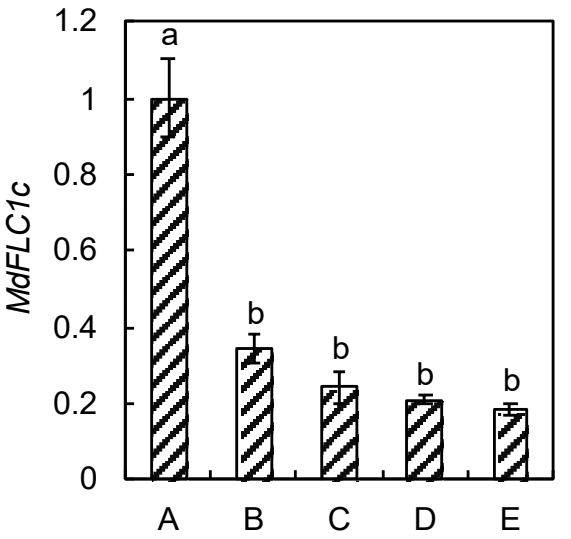

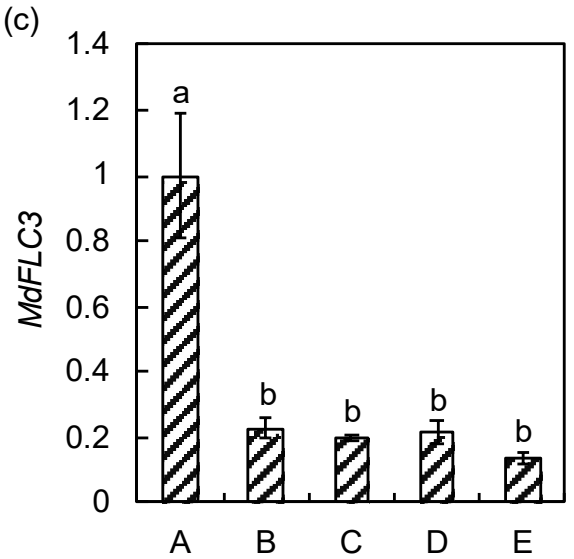

Figure 4. Changes in the expression levels of $M d F L C 1 b$ (a), $M d F L C 1 c$ (b), and $M d F L C 3$ (c) during phase transition in apple seedlings. The expression level of MdFLC1a was undetectable. Total RNA was prepared from the juvenile phase (A), transition phase (B and C), and adult phase (D and E) in early July 2009 as described in [27]. Relative expression was determined in triplicate measurements taken from three independent biological replicates. The relative expression levels were normalized against $M d A C T I N$ with standard errors, and the maximum level of the transcripts was set at 1.0. The values with different letters for each gene significantly differed between positions at $p<0.05$ according to a Tukey test.

2.4. Transformation of Arabidopsis with MdFLC3 cDNA

Delays in bolting were observed in more than three transgenic plants from individual seeds $\left(\mathrm{T}_{1}\right)$ obtained by Agrobacterium in plant vacuum infiltration transformation. Detailed analysis was performed on their progenies, lines FOX1 and FOX2. The growth of FOX1 and FOX2 lines was observed and expression analysis of AtFT was then performed (Figure 5; Figure S1a,b, Supplementary Materials). The number of days from sowing to bolting was 31.8 in the wild-type (WT) control, and that in FOX1 and FOX 2 was 35.5 and 35.7 days, respectively. The number of rosette leaves at the time of bolting was 14.4 in the wild-type and 17.5 and 18.2 in FOX1 and FOX2, respectively. A lower expression level of AtFT was observed with late flowering in the FOX lines. A very high expression level of MdFLC3 was confirmed in the FOX lines, whereas only a trace expression of endogenous FLC (AtFLC) was found in the FOX lines and wild-type (Figure S2, Supplementary Materials). The average value of transformants derived from individual seeds, which are different from FOX 1 and FOX 1 lines, at the beginning of transformation showed delayed bolting, supporting the above result (Figure S1c,d). 
(a)

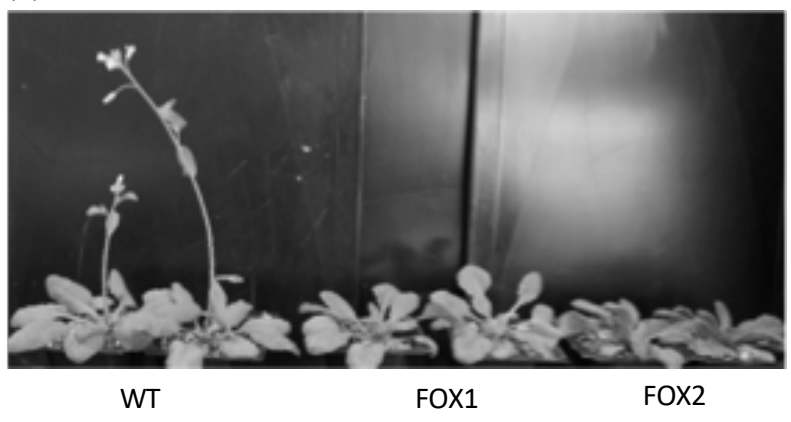

(c) 20

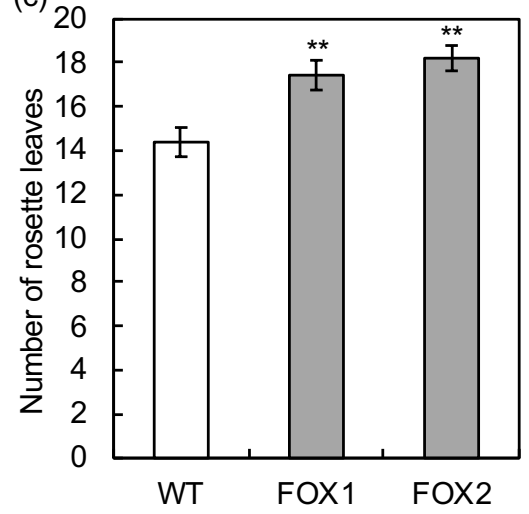

(b)

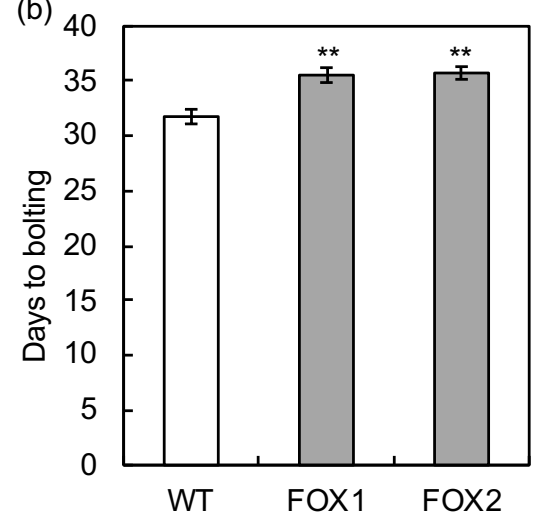

(d)

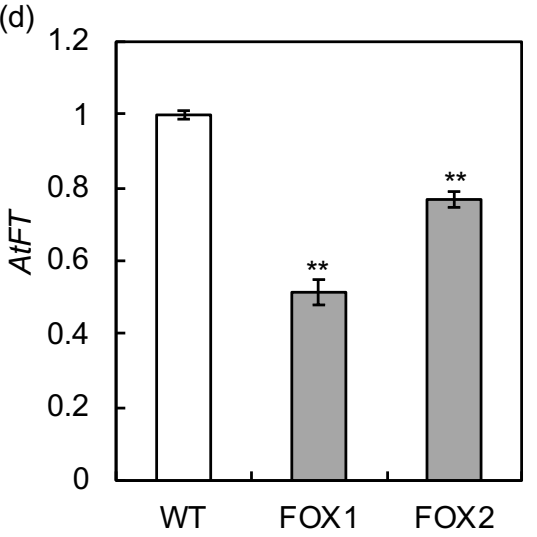

Figure 5. The overexpression of $M d F L C 3$ and phenotypic analysis in Arabidopsis. Flowering phenotypes 32 days after sowing (a), days to bolting from sowing $(\mathbf{b})$, the number of rosette leaves at bolting time (c), and the expression levels of AtFT (d) in the MdFLC3 transgenic (FOX1, 2) and wild-type (WT) plants grown under a $16 \mathrm{~h}$ photoperiod. The values with ** significantly differed between FOX and WT plants at $p<0.01$, according to the Dunnett test. The values in $(\mathbf{b}, \mathbf{c})$ indicate means and standard errors ( $\mathrm{n}=30$ or 31$)$. The relative expression of AtFT was determined from triplicate measurements of three independent biological replicates 15 days after sowing (d). The relative expression levels were normalized against AtACTIN with standard errors and the maximum level of the transcripts was set at 1.0 .

\section{Discussion}

A phylogenetic tree analysis, including other MADS-box genes such as SVP and SOC1, revealed that both MdFLC1 and MdFLC3 belong to the same FLC group as VvFLC [30] and PtFLC [26], which has been reported to function as a floral repressor (Figure 2). Therefore, MdFLC1 and MdFLC3 were further investigated as apple FLCs in this study. FLC is one of the MADS-box proteins, which are transcription factors having a highly conserved region of approximately 60 amino acids, MADS-box, that is involved in DNA binding and dimer formation. Many MADS-box proteins in plants are classified as MIKC-type, contain MADS-, I-, K-, and C-domains [31], and form dimers and higher multimers to function [32,33]. MdFLC1c and MdFLC3 contained these four conserved domains.

Three cDNA sequences of MdFLC1 were searched in the GDR database, and MdFLC1 was found to correspond to MD05G1037100. MdFLC1c mRNA contained all exons, whereas MdFLC1a and MdFLC1b mRNA contained the sequences of the fourth and third introns, which were not removed in splicing, respectively (Figure 6). Therefore, the deduced amino acid sequence of MdFLC1c contained MADS-, I-, $\mathrm{K}-$, and C-domains, whereas the sequences of MdFLC1a and MdFLC1b lacked K- and C-domains because of the stop codons in the intron sequences of their mRNA (Figure 1). K- and C-domains are important for protein-protein interactions and other functions in MIKC-type MADS proteins [34]. These results suggest that MdFLC1c plays the role of $M d F L C 1$ and that $M d F L C 1 a$ and $M d F L C 1 b$ are 
expected to be non-functional. However, since the regulation of expression by selective splicing has been reported in plant response to environmental stress [35], expression analysis was performed in the three splicing variants of $M d F L C 1$. The $M d F L C 3$ cDNA sequence was consistent with that of MD10G1041100 in the GDR database, and its deduced amino acid sequence contained the four domains of an MIKC-type MADS protein (Figure 1), suggesting that MdFLC3 functions as an MIKC-type MADS protein.
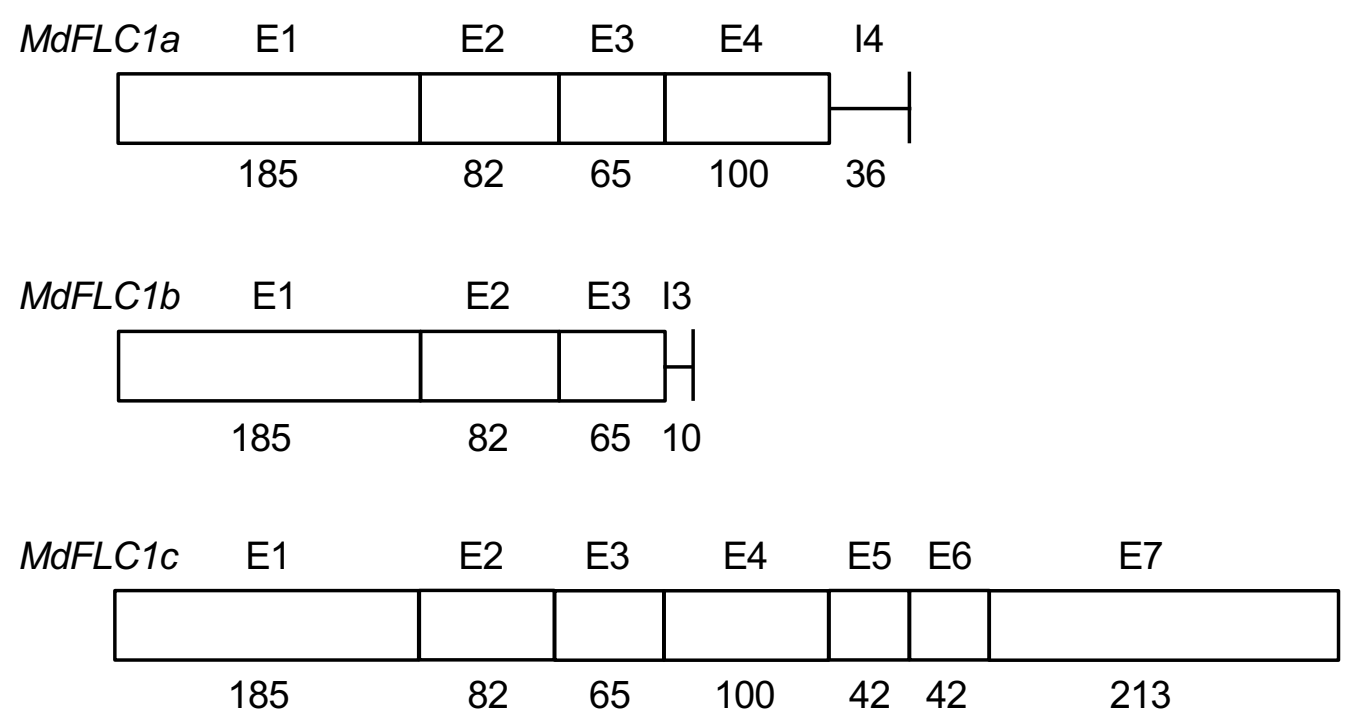

Figure 6. Transcript structures of MdFLC1 splice variants, MdFLC1a, MdFLC1b, and MdFLC1c. E and I with numbers indicate exons and introns, respectively. Numbers under the bar correspond to their sizes, in base pairs.

In the present study, the expression levels of MdFLC1a, MdFLC1b, and MdFLC1c decreased during the period of flowering induction in a seasonal expression pattern in the adult trees. In the annual growth cycle of apple, MdFT1, which is a floral integrator, shows high expression, and the expression level of $M d T F L$, which is a floral repressor, decreases during the period of flowering induction $[9,27]$. Therefore, the expression of MdFLC1a, MdFLC1b, and particularly MdFLC1c, which is expected to translate functional proteins, is likely involved in suppression of flowering in the annual growth cycle of adult apple trees, as is MdTFL. In contrast, the expression level of MdFLC3 did not decrease during the period of flowering induction, suggesting that MdFLC3 is not involved in flowering induction in the annual growth cycle of adult trees.

Because phase change is observable in individuals, seedlings can be used for expression analysis of phase transition [36]. Five sites in the seedlings used in this study show phase transition based on juvenile characteristics such as flower bud formation, leaf size, and leaf serration, and were used for $M d F L C$ expression analysis [27]. Although expression of MdFLC1a was not detected, the expression levels of $M d F L C 1 b$ and $M d F L C 1 c$ were high in the juvenile phase and low in the transitional and adult phases. The expression level of MdFLC3 was similarly high in the juvenile phase. The expression pattern of MdFLC3 suggests that it does not play a role in flowering in the annual growth cycle but acts specifically in juvenility. A similar seasonal expression pattern was observed in MdFLC3 within buds in the adult trees and confirmed the specific role of MdFLC3 (Figure S3, Supplementary Materials). Furthermore, the expression of $M d F L C 1 c$ in the juvenile phase of seedlings is significantly lower than the expression in the adult trees (approximately 1/6, data not shown). Therefore, we focused on this specific role of $M d F L C 3$ and subjected $M d F L C 3$ to functional analysis by the transformation of Arabidopsis. The results showed that $M d F L C 3$ is a floral repressor, confirming its role in the juvenility of apple. Since the result for phase transition was obtained in crossed seedlings, a genotype-specific effect 
could not be excluded. Further study using other several genotype combinations and apomixis will be necessary to confirm the findings of the present study.

FLC suppresses the expression of FT containing the CArG box, which is an FLC-binding sequence, in the promoter region and first intron in Arabidopsis leaves [17]. Apples also have sufficient genomic information and such binding sites can be analyzed. Since the MdFTs in the database have CArG box-like sequences in the intron and promoter region (Table 1), it can be expected that MdFLC1 and MdFLC3 bind to these sequences to control annual flowering and juvenility. Regarding the molecular mechanism of juvenility of woody plants, including fruit trees, little is known about genes located upstream of the flowering pathway. Our results suggest that the apple homolog of $F L C$, which appears to play a central role relatively upstream of the flowering pathway, could be involved in juvenility as well as in annual flowering. Testing the correlation between MdFLC characteristics and the length of the juvenile phase could provide valuable insight into the function of MdFLC in the regulation of this process in the near future. In addition, if this gene could be used as a marker, it would be possible to breed cultivars with a short juvenile period as well as various useful traits by marker-assisted selection [21,37]. As apples have advanced in genome information and genome editing technology [38], accumulating results as a model will be utilized for other fruit trees.

Table 1. Putative CArG boxes in $M d F T^{\mathrm{a}}$.

\begin{tabular}{cllcc}
\hline Gene & $\begin{array}{c}\text { Accession } \\
\text { Number }\end{array}$ & \multicolumn{1}{c}{ Putative CArG Box } & Strand & $\begin{array}{c}\text { Position of 1st C } \\
\text { from ATG }\end{array}$ \\
\hline \multirow{2}{*}{ MdFT1 } & AB458506 & AACTCCATTAATTGCAGG & Top & +289 \\
& & TACTCCTTATTTTGTCAA & Top & +846 \\
MdFT2 & AB458504 & CTAACCATTAATTGTGTT & Top & +1001 \\
& & AGATCCTAAAAAAGTATA & Bottom & +994 \\
& & GTATCCAAATAAGTTGC & Bottom & -159 \\
& GTCTCCTAATTTGTTGT & Bottom & -745 \\
\hline
\end{tabular}

a The boxes in the introns and promoter regions, the upstream 1500-bp MdFT promoter sequences of the start codon were checked in both strands according to Helliwell et al. [17]. ${ }^{\mathrm{b}}$ The boxes in the introns and the promoter regions are shown with the positive and negative number of positions, respectively.

\section{Materials and Methods}

\subsection{Plant Materials}

Mature leaves in the juvenile phase of 8-year-old apple (Malus domestica) seedlings of a cross between 'Fuji' and 'Himekami' were used for cDNA isolation and sequence analysis of MdFLC. Mature leaves from 11-year-old apple trees ('Fuji' grafted onto M.9, M. prunifolia) were used as adult trees. For expression analysis in adult trees, mature leaves were sampled from 20-year-old apple trees ('Fuji' grafted onto M.9, M. prunifolia) on May 1, June 1, July 1, and August 1, 2016. For expression analysis of phase transition, mature leaves were collected from the juvenile to adult phase in 8-year-old apple seedlings of a cross between 'Fuji' and 'Himekami' [27]. All samples were collected in the Tohoku University (Sendai, Japan) experimental field at $38^{\circ} 16^{\prime} \mathrm{N}$ and $140^{\circ} 52^{\prime} \mathrm{E}$.

\section{2. cDNA Isolation, Sequence Analysis, and Phylogenetic Tree Analysis of MdFLC}

To isolate MdFLC1 cDNA, the apple EST sequences were searched using DDBJ and GDR. DDBJ was also used to search homologous genes in other plants. The cetyltrimethylammonium bromide (CTAB) method [27] and a TaKaRa RNA PCR kit (AMV) (Takara-Bio, Kusatsu, Japan) were used for RNA extraction and reverse transcription, respectively. Cloning of MdFLC3 CDNA was performed by PCR with degenerate primers MdMADSF and MdMADSR (Table S1, Supplementary Materials) based on highly conserved sequences in the MADS-box protein using PROSITE (https://prosite.expasy.org). The restriction sites of $E c o R I$ and BamHI were added to the primers in advance. PCR was performed with cDNA from leaves in the juvenile and adult phases. PCR products were electrophoresed on 
agarose gel, and amplified fragments of the expected size were collected using TaKaRa RECOCHIP (TaKaRa). pUC18 was digested with EcoRI and BamHI, and the PCR fragments were ligated into this vector and then transformed into Escherichia coli. The plasmid was purified for sequence analysis. Sequence Alignment by ClustalW (http://align.genome.jp) was used to prepare the sequence alignments and phylogenetic trees.

\subsection{Expression Analysis by Real-Time PCR}

For expression analysis in the adult trees, RNA extraction and reverse transcription were performed using a Cica Geneus RNA prep kit for Plant (Kanto Kagaku, Tokyo, Japan) and ReverTra Ase qPCR RT Master Mix with gDNA Remover (TOYOBO, Osaka, Japan), respectively. THUNDERBIRD SYBR qPCR Mix (TOYOBO) was used for the subsequent PCR. For expression analysis with phase transition, RNA extraction and reverse transcription were performed using the CTAB method [27] and QuantiTect Reverse Transcription Kit (Qiagen, Hilden, Germany), respectively. A QuantiTect SYBR Green PCR Kit (Qiagen) was used for the subsequent PCR. Real-time PCR was performed as described in Ikeda et al. [39], using the primer sets listed in Table S1. Coefficients of variant for quantification cycle of the reference genes among samples were $1.85 \%, 2.75 \%$, and $1.01 \%$ in Figures 3-5, respectively.

\subsection{Transformation of Arabidopsis with MdFLC3 cDNA}

The translated region of MdFLC3 cDNA was amplified by PCR using Q5 DNA polymerase (New England Biolabs, Ipswich,, MA, USA) electrophoresed in an agarose gel. It was then collected from the gel and used as an insert. The primers used were MdFLC3InsertF and MdFLC3InsertR, in which BamHI and SacI sites were added to the $5^{\prime}$ and $3^{\prime}$ ends, respectively (Table S2, Supplementary Materials). The GUS sequence in the binary vector pBI121 was excised using BamHI and SacI, and the MdFLC3 insert was introduced into the vector instead. The resultant vector containing MdFLC3 cDNA with CaMV $35 S$ promoter was used for the transformation of Arabidopsis thaliana ecotype Columbia (Inplanta Innovation Inc., Yokohama, Japan). Transformed seeds $\left(\mathrm{T}_{1}\right)$ were selected in a medium containing kanamycin, and a transgene check was performed by PCR using the primer set for MdFLC3 (Table S2). Expression analysis was performed by real-time PCR using the primer sets for AtFT, MdFLC3, and AtFLC, and the Arabidopsis actin primer was set as a reference (Table $\mathrm{S} 2$ ). Seeds $\left(\mathrm{T}_{2}\right)$ were collected separately from individuals $\left(\mathrm{T}_{1}\right)$ derived from individual seeds initially obtained by Agrobacterium in planta vacuum infiltration transformation and each of them was sown as a line. In the $\mathrm{T}_{2}$ generation, segregation of the transgene was checked in each line and seeds $\left(\mathrm{T}_{3}\right)$ were collected. Homozygous seeds that did not segregate in $\mathrm{T}_{3}$ were used. The homozygous seeds $\left(\mathrm{T}_{3}\right)$ were planted and grown in vermiculite/pearlite $(1: 1)$ at $22{ }^{\circ} \mathrm{C}$ under a $16 \mathrm{~h}$ photoperiod, and the number of rosette leaves and days after sowing was measured at bolting [40].

Supplementary Materials: Supplementary materials can be found at http://www.mdpi.com/1422-0067/21/12/ 4562/s1. Table S1. Primers used for cloning of cDNA encoding MdFLC and real-time PCR in apple; Table S2. Primers used for transformation of Arabidopsis with MdFLC3 cDNA and real-time PCR in transgenic Arabidopsis; Figure S1. Phenotype analysis in the MdFLC3 transgenic (FOX) and wild-type (WT) plants as support data for Figure 5; Figure S2. Expression levels of MdFLC3 and AtFLC in the MdFLC3 transgenic (FOX1, 2) and wild-type (WT) plants; Figure S3. Seasonal changes in the expression levels of MdFLC3 in the buds of adult trees.

Author Contributions: Conceptualization, S.K. and Y.K.; methodology, H.K., T.S., and S.K.; investigation, H.K. and N.I.; formal analysis, H.K., N.I., and T.S.; resources, K.K.; data curation, K.K.; writing-original draft preparation, H.K.; writing-review and editing, K.K. and Y.K.; visualization, H.K.; supervision, S.K. and Y.K.; project administration, Y.K.; funding acquisition, K.K. and Y.K. All authors have read and agreed to the published version of the manuscript.

Funding: This research was supported by Grants-in-Aid for Scientific Research (24248006), Japan.

Acknowledgments: The authors thank Shohei Hano and Mai Asakawa for preliminary experiments and materials used for experiments.

Conflicts of Interest: The authors declare no conflict of interest. 


\section{Abbreviations}

$\begin{array}{ll}\text { FLC } & \text { FLOWERING LOCUS C } \\ \text { TFL1 } & \text { TERMINAL FLOWER 1 } \\ \text { FT } & \text { FLOWERING LOCUS T } \\ \text { LFY } & \text { LEAFY } \\ \text { AP1 } & \text { APETALA1 } \\ \text { EST } & \text { expressed sequence tag } \\ \text { DDBJ } & \text { DNA Data Bank of Japan } \\ \text { WT } & \text { wild-type }\end{array}$

\section{References}

1. Zimmerman, R.H. Juvenility and flowering in woody plants: A review. HortScience 1972, 7, 447-455.

2. Visser, T. Juvenile phase and growth of apple and pear seedlings. Euphytica 1964, 13, 119-129.

3. Visser, T. The effect of rootstocks on growth and flowering of apple seedlings. J. Am. Soc. Hortic. Sci. 1973, 98, 26-28.

4. Hackett, W.P. Juvenility, maturation, and rejuvenation in woody plants. Hortic. Rev. 1985, 7, 109-155.

5. Velasco, R.; Zharkikh, A.; Affourtit, J.; Dhingra, A.; Cestaro, A.; Kalyanaraman, A.; Fontana, P.; Bhatnagar, S.K.; Troggio, M.; Pruss, D.; et al. The genome of the domesticated apple (Malus $\times$ domestica Borkh.). Nat Genet. 2010, 42, 833-839. [CrossRef]

6. Eccher, G.; Ferrero, S.; Populin, F.; Colombo, L.; Botton, A. Apple (Malus domestica L. Borkh) as an emerging model for fruit development. Plant Biosyst. 2014, 148, 157-168. [CrossRef]

7. He, P.; Li, L.G.; Li, H.F.; Wang, H.B.; Yang, J.M. Development and application S-SAP molecular markers for the identification of apple (Malus Domestica Borkh.) sports. J. Hortic. Sci. Biotechnol. 2015, 90, $297-302$. [CrossRef]

8. Poething, R.S. Phase change and the regulation of shoot morphogenesis in plants. Science 1990, 250, 923-930. [CrossRef]

9. Mimida, N.; Komori, S.; Suzuki, A.; Wada, M. Functions of the apple TFL1/FT orthologs in phase transition. Sci. Hortic. 2013, 156, 106-112. [CrossRef]

10. Kotoda, N.; Wada, M.; Kusaba, S.; Kano-Murakami, Y.; Masuda, T.; Soejima, J. Overexpression of MdMADS5, an APETALA1-like gene of apple, causes early flowering in transgenic Arabidopsis. Plant Sci. 2002, 162, 679-687. [CrossRef]

11. Kotoda, N.; Wada, M. MdTFL1, a TFL1-like gene of apple retards the transition from the vegetative to reproductive phase in transgenic Arabidopsis. Plant Sci. 2005, 168, 95-104. [CrossRef]

12. Wada, M.; Cao, Q.; Kotoda, N.; Soejima, J.; Masuda, T. Apple has two orthologues of FLORICAULA/LEAFY involved in flowering. Plant Mol. Biol. 2002, 49, 567-577. [CrossRef] [PubMed]

13. Pena, L.; Martin-Trillo, M.; Juarez, J.; Pina, J.A.; Navarro, L.; Martinez-Zapater, J.M. Constitutive expression of Arabidopsis LFAFY or APETALA1 gene in citrus reduces their generation time. Nat. Biotech. 2001, 19, 263-267. [CrossRef]

14. Zhang, J.Z.; Mei, L.; Liu, R.; Khan, M.R.G.; Hu, C.G. Possible involvement of locus-specific methylation on expression regulation of $L E A F Y$ homologous gene (CiLFY) during precocious trifoliate orange phase change process. PLoS ONE 2014, 9, e88558. [CrossRef] [PubMed]

15. Srikanth, A.; Schmid, M. Regulation of flowering time: All roads lead to Rome. Cell Mol. Life Sci. 2011, 68, 2013-2037. [CrossRef] [PubMed]

16. Michaels, S.D.; Amasino, R.M. FLOWERING LOCUS C encodes a novel MADS domain protein that acts as a repressor of flowering. Plant Cell 1990, 11, 949-956. [CrossRef] [PubMed]

17. Helliwell, C.A.; Wood, C.C.; Robertson, M.; James, P.W.; Dennis, E.S. The Arabidopsis FLC protein interacts directly in vivo with SOC1 and FT chromatin and is part of a high-molecular-weight protein complex. Plant J. 2006, 46, 183-192. [CrossRef] 
18. Hepworth, S.R.; Valverde, F.; Ravenscroft, D.; Mouradov, A.; Coupland, G. Antagonistic regulation of flowering-time gene SOC1 by CONSTANS and FLC via separate promoter motifs. EMBO J. 2002, 21, 4327-4337. [CrossRef]

19. Searle, I.; He, Y.; Turck, F.; Vincent, C.; Fornara, F.; Krober, S.; Amasino, R.A.; Coupland, G. The transcription factor $F L C$ confers a flowering response to vernalization by repressing meristem competence and systemic signaling in Arabidopsis. Genes Dev. 2006, 20, 898-912. [CrossRef]

20. Kumar, G.; Arya, P.; Gupta, K.; Randhawa, V.; Achaya, V.; Singh, A.K. Comparative phylogenetic analysis and transcriptional profiling of MADS-box gene family identified DAM and FLC-like genes in apple (Malus x domestica). Sci. Rep. 2016, 6, 20695. [CrossRef]

21. Urrestarazu, J.; Muranty, H.; Denancé, C.; Leforestier, D.; Ravon, E.; Guyader, A.; Guisnel, R.; Feugey, L.; Aubourg, S.; Celton, J.-M.; et al. Genome-wide association mapping of flowering and ripening periods in apple. Front. Plant Sci. 2017, 8, 1923. [CrossRef] [PubMed]

22. Porto, D.D.; Bruneau, M.; Perini, P.; Anzanello, R.; Jean-Pierre, R.; Dos Santos, H.P.; Fialho, F.B.; Revers, L.F. Transcription profiling of the chilling requirement for bud break in apples: A putative role for FLC-like genes. J. Exp. Bot. 2015, 66, 2659-2672. [CrossRef] [PubMed]

23. Zong, X.; Zhang, Y.; Walworth, A.; Tomaszewski, E.M.; Callow, P.; Zhong, G.Y.; Song, G.Q. Constitutive expression of an apple FLC3-like gene promotes flowering in transgenic blueberry under nonchilling conditions. Int. J. Mol. Sci. 2019, 20, 2775. [CrossRef] [PubMed]

24. Wells, E.C.; Vendramin, E.; Tarodo, J.S.; Verge, I.; Bielenberg, G.D. A genome-wide analysis of MADS-box genes in peach [Prunus persica (L.) Batsch]. BMC Plant Biol. 2015, 15, 41. [CrossRef]

25. Xu, Z.; Zhang, Q.; Sun, L.; Du, D.; Cheng, T.; Pan, H.; Yang, W.; Wang, J. Genome-wide identification, characterization and expression analysis of the MADS-box gene family in Prunus mume. Mol. Genet. Genom. 2014, 289, 903-920. [CrossRef]

26. Zhang, J.Z.; Li, Z.M.; Mei, L.; Yao, J.L.; Hu, C.G. PtFLC homolog from trifoliate orange (Poncirus trifoliate) is regulated by alternative splicing and experiences seasonal fluctuation in expression level. Planta 2009, 229, 847-859. [CrossRef]

27. Hatsuda, Y.; Nishio, S.; Komori, S.; Nishiyama, M.; Kanahama, K.; Kanayama, Y. Relationship between MdMADS11 gene expression and juvenility in apple. J. Soc. Hortic. Sci. 2011, 80, 396-403. [CrossRef]

28. Zeevaart, J.A.D. Leaf-produced floral signals. Curr. Opin. Plant Biol. 2008, 11, 541-547. [CrossRef]

29. Freiman, A.; Golobovitch, S.; Yablovitz, Z.; Belausov, E.; Dahan, Y.; Peer, R.; Avraham, L.; Freiman, Z.; Evenor, D.; Reuveni, M.; et al. Expression of flowering locus T2 transgene from Pyrus communis L. delays dormancy and leaf senescence in Malus x domestica Borkh, and causes early flowering in tobacco. Plant Sci. 2015, 241, 164-176. [CrossRef]

30. Díaz-Riquelme, J.; Martínez-Zapater, J.M.; Carmona, M.J. Transcriptional analysis of tendril and inflorescence development in grapevine. PLoS ONE 2014, 9, e92339. [CrossRef]

31. De Bodt, S.; Raes, J.; Florquin, K.; Rombauts, S.; Rouzé, P.; Theissen, G.; Van de Peer, Y. Genomewide structural annotation and evolutionary analysis of the type I MADS-box genes in plants. J. Mol. Evol. 2003, 56, 573-586. [CrossRef]

32. Theissen, G.; Saedler, H. Floral quartets. Nature 2001, 409, 469-471. [CrossRef]

33. Honma, T.; Goto, K. Complexes of MADS-box proteins are sufficient to convert leaves into floral organs. Nature 2001, 409, 525-529. [CrossRef] [PubMed]

34. Kaufmann, K.; Melzer, R.; Theissen, G. MIKC-type MADS-domain proteins: Structural modularity, protein interactions and network evolution in land plants. Gene 2005, 347, 183-198. [CrossRef] [PubMed]

35. Shang, X.; Cao, Y.; Ma, L. Alternative splicing in plant genes: A means of regulating the environmental fitness of plants. Int. J. Mol. Sci. 2017, 18, 432. [CrossRef] [PubMed]

36. Carlsbecker, A.; Tandre, K.; Johanson, U.; Englund, M.; Engstrom, P. The MADS-box gene DAL1 is a potential mediator of the juvenile-to-adult transition in Norway spruce (Picea abies). Plant J. 2004, 40, 546-557. [CrossRef]

37. Moriya, S.; Terakami, S.; Okada, K.; Shimizu, T.; Adachi, Y.; Katayose, Y.; Fujisawa, H.; Wu, J.; Kanamori, H.; Yamamoto, T.; et al. Identification of candidate genes responsible for the susceptibility of apple (Malus $\times$ domestica Borkh.) to Alternaria blotch. BMC Plant Biol. 2019, 19, 132. [CrossRef]

38. Zhou, J.; Li, D.; Wang, G.; Wang, F.; Kunjal, M.; Joldersma, D.; Liu, Z. Application and future perspective of CRISPR/Cas9 genome editing in fruit crops. J. Integr. Plant Biol. 2020, 62, 269-286. [CrossRef] 
39. Ikeda, H.; Shibuya, T.; Imanishi, S.; Aso, H.; Nishiyama, M.; Kanayama, Y. Dynamic Metabolic regulation by a chromosome segment from a wild relative during fruit development in a tomato introgression line, IL8-3. Plant Cell Physiol. 2016, 57, 1257-1270. [CrossRef]

40. Kanayama, Y.; Mizutani, R.; Yaguchi, S.; Hojo, A.; Ikeda, H.; Nishiyama, M.; Kanahama, K. Characterization of an uncharacterized aldo-keto reductase gene from peach and its role in abiotic stress tolerance. Phytochemistry 2014, 104, 30-36. [CrossRef]

(C) 2020 by the authors. Licensee MDPI, Basel, Switzerland. This article is an open access article distributed under the terms and conditions of the Creative Commons Attribution (CC BY) license (http://creativecommons.org/licenses/by/4.0/). 